\title{
The Spinal Interneurons and Properties of Glutamatergic Synapses in a Primitive Vertebrate Cutaneous Flexion Reflex
}

\author{
Wen-Chang Li, Stephen R. Soffe, and Alan Roberts \\ School of Biological Sciences, University of Bristol, Bristol, BS8 1UG, United Kingdom
}

\begin{abstract}
Unlike the monosynaptic "stretch" reflex, the exact neuronal pathway for a simple cutaneous reflex has not yet been defined in any vertebrate. In young frog tadpoles, we made whole-cell recordings from pairs of spinal neurons. We found direct, excitatory, glutamatergic synapses from touch-sensitive skin-sensory neurons to sensory pathway interneurons, and then from these sensory interneurons to motoneurons and premotor interneurons on the other side of the body. We conclude that the minimal pathway for this primitive reflex, in which stroking the skin on one side leads to flexion on the other side, is disynaptic. This detailed circuit information has allowed us to ask whether the properties of glutamatergic synapses during the first day of CNS development are tuned to their function in the tadpole's responses. Stroking the skin excites a few sensory neurons. These activate primarily AMPA receptors producing short, strong excitation that activates many sensory pathway interneurons but only allows temporal summation of closely synchronous inputs. In contrast, the excitation produced in contralateral neurons by the sensory pathway interneurons is weak and primarily mediated by NMDA receptors. As a result, considerable summation is required for this excitation to lead to postsynaptic neuron firing and a contralateral flexion. We conclude that from their early functioning, synapses from sensory neurons are strong and those from sensory pathway interneurons are weak. The distribution of glutamate receptors at synapses in this developing circuit is tuned so that synapses have properties suited to their roles in the whole animal's reflex responses.
\end{abstract}

Key words: locomotion; reflex; spinal cord; NMDA; glutamate receptors; Xenopus

\section{Introduction}

This study addresses one main question. How is the primitive vertebrate spinal flexion reflex organized? Although most textbooks of neuroscience show the mammal flexion reflex with two layers of interneurons in the pathway, we know of no case in the vertebrates in which the spinal pathway from a skin stimulus to a motor response can be traced, synapse by synapse, from the sensory receptors via defined interneurons to the motoneurons $(\mathrm{mn})$. It also sheds some light on a second question: what happens when central glutamatergic synapses develop in such a pathway? Do they start as "silent" synapses with just NMDA receptors (NMDARs), only later acquiring AMPA receptors (AMPARs) and becoming functional as a result of activity (Feldman and Knudsen, 1998; Zhu and Malinow, 2002), or, are synapse properties activity independent and tuned to their circuit functions from an early stage (Renger et al., 2001; Ziv and Garner, 2001)? We examined these questions by exploiting the frog tadpole spinal cord in which there are few interneuron types (Roberts et al., 2000; Li et al., 2001), and whole-cell recordings can be made from pairs of neurons to look at synaptic interactions (Li et al., 2002).

In hatchling Xenopus tadpoles, stroking the skin on one side of the trunk can lead to a flexion on the opposite side followed by swimming away (Boothby and Roberts, 1995). Can we trace the

Received June 20, 2003; revised Aug. 1, 2003; accepted Aug. 3, 2003.

We thank Tim Colborn, Derek Dunn, and Bob Porter for technical assistance and the Wellcome Trust for support. Correspondence should be addressed to Dr. Wen-Chang Li, School of Biological Sciences, University of Bristol, Woodland Road, Bristol, BS8 1UG, UK. E-mail: wenchang.li@bristol.ac.uk.

Copyright $\odot 2003$ Society for Neuroscience $\quad$ 0270-6474/03/239068-10\$15.00/0 cellular components of this response pathway? The trunk skin is innervated by one class of sensory neurons in the dorsal spinal cord, Rohon-Beard (RB) neurons. These respond to touch and strokes, adapt very rapidly, and can also be excited by current pulses to the skin (Clarke et al., 1984). In the dorsolateral spinal cord, there are sensory pathway dorsolateral commissural interneurons (dlc) with dendrites in positions to contact the longitudinal central axons of Rohon-Beard neurons. These dlc interneurons have axons that project to the opposite side of the cord. Intracellular recordings with dye injection have shown that they are excited at short latency after touch or current pulses to the skin; thus, they are presumed to be excited directly by the axons of the sensory RB neurons (Clarke et al., 1984; Roberts and Sillar, 1990). Recordings from unidentified spinal interneurons have shown that $\mathrm{RB}$ neurons release a transmitter, presumed to be glutamate, that activates NMDARs and non-NMDARs to produce strong excitation (Sillar and Roberts, 1988a). After skin stimulation, unidentified ventral neurons on the opposite side of the spinal cord that are rhythmically active during swimming receive excitation. Indirect evidence supports the proposal that this excitation comes from dlc interneurons, is glutamatemediated, and activates NMDARs (Roberts and Sillar, 1990).

Our aim was to use paired recordings with dye injection to confirm the identity of the sensory pathway interneurons excited by sensory RB neurons and to find which neurons the sensory pathway interneurons themselves excited on the opposite side. Using this detailed circuit information, we asked whether the properties of glutamatergic synapses between these neurons are 
homogeneous at this early stage of CNS development or whether they are tuned to their functions in this simple reflex pathway.

\section{Materials and Methods}

Details of the methods have been given recently (Li et al., 2002). In brief, Xenopus tadpoles at stage 37/38 (see Fig. 2A) were anesthetized with $0.1 \%$ MS-222 (3-aminobenzoic acid ester; Sigma, Poole, UK) and then pinned in a small bath of saline including the following (in $\mathrm{mM}$ ): 115 $\mathrm{NaCl}, 3 \mathrm{KCl}, 2 \mathrm{CaCl}_{2}, 2.4 \mathrm{NaHCO}_{3}, 1 \mathrm{MgCl}_{2}, 10 \mathrm{HEPES}$, adjusted with 5 $\mathrm{M} \mathrm{NaOH}$ to $\mathrm{pH}$ 7.4. In many paired recording experiments, $1 \mathrm{~mm} \mathrm{MgCl}_{2}$ was replaced by $1 \mathrm{~mm} \mathrm{CaCl}_{2}$. The dorsal fin was cut, and the tadpole was transferred to $10 \mu \mathrm{M} \alpha$-bungarotoxin saline. After immobilization, the tadpole was repinned so that skin and muscles over the right side of the spinal cord could be removed. A dorsal cut was then made along the midline of the spinal cord to open the neurocoel and expose neuronal cell bodies. Additional small cuts were made to expose more ventral neurons. In experiments on transmission from dlc interneurons to contralateral rhythmic neurons, the right side of the spinal cord in segments $3-5$ was removed to allow better illumination to see and access left-side neurons more easily. The animal was then repinned on a small rotating Sylgard stage in a $700 \mu \mathrm{l}$ recording chamber that allowed bright-field illumination from below on an upright Nikon (Tokyo, Japan) E600FN microscope. The animal was tilted to an angle, which allowed the exposed neuronal cell bodies on the left and right sides of the cord to be seen using a $40 \times$ water immersion lens. Saline in the chamber was circulated at $\sim 2$ $\mathrm{ml} / \mathrm{min}$. Drops of antagonists were added to a $100 \mu \mathrm{l}$ chamber upstream to the recording chamber (bath application) or applied close to the recorded neuron soma by applying gentle pressure to the solution in a pipette with a tip diameter of $20-30 \mu \mathrm{m}$ (microperfusion). Unless specified, the concentrations for 1,2,3,4-tetrahydro-6-nitro-2,3-dioxobenzo[f] quinoxaline-7-sulfonamide (NBQX), D-2-amino-5-phosphonopentanoic acid (D-AP-5), and $\mathrm{Mg}^{2+}$ used in this study were $5 \mu \mathrm{m}, 50$ $\mu \mathrm{m}$, and $1 \mathrm{~mm}$, respectively. NBQX and D-AP-5 were obtained from Tocris Cookson (Bristol, UK); mecamylamine and strychnine were obtained from Sigma.

Extracellular recordings of ventral root activity were made using glass suction electrodes placed against the intermyotome clefts. A stimulating suction electrode was placed on the head or tail skin to start fictive swimming activity. Patch pipettes were filled with $0.1 \%$ neurobiotin in intracellular solution (100 mM K-gluconate, $2 \mathrm{~mm} \mathrm{MgCl}_{2}, 10 \mathrm{~mm}$ EGTA, 10 mM HEPES, $3 \mathrm{~mm} \mathrm{Na} \mathrm{Na}_{2} \mathrm{ATP}, 0.5 \mathrm{~mm} \mathrm{NaGTP}$, adjusted to $\mathrm{pH} 7.3$ with $\mathrm{KOH})$ and had resistances of $\sim 10 \mathrm{M} \Omega$. Patch pipettes were advanced under visual control to contact exposed neuron somata. Positive pressure (5-20 $\mathrm{cm} \mathrm{H}_{2} \mathrm{O}$ ) was always applied to the pipette solution before trying to get a seal. Signals were recorded with an Axoclamp 2B in "conventional" bridge or continuous single-electrode voltage-clamp mode. Data were acquired with Signal software through a CED 1401 Plus (Cambridge Electronics Design, Cambridge, UK) with a sampling rate of $10 \mathrm{kHz}$. Stimuli to the skin were controlled using the CED 1401 Plus configured by Signal and given via an optically coupled isolator. Off-line analyses were made with Minitab and Excel (Microsoft, Seattle, WA). All data were tested for normality (Anderson-Darling). Median values are given for non-normal data; otherwise, all values are given as mean \pm SD.

In patch-pipette recordings from pairs of neurons, current-induced spikes in one cell caused a small cross-talk artifact in the other (Li et al., 2002). These artifacts were sometimes removed from recordings in which they obscured the onset of small EPSPs evoked by current-induced spikes. To do this, averaged records of EPSP failures (artifact alone) were subtracted from EPSP records (EPSP plus artifact).

Once physiological testing was completed, trains of positive-current pulses (10-100 pA; 500 msec duration) were applied for 2-5 min to label recorded neurons with neurobiotin. The tadpoles were then left for 30 min before fixing in $2 \%$ glutaraldehyde in $0.1 \mathrm{~m}$ phosphate buffer, $\mathrm{pH} 7.2$, at $\sim 4^{\circ} \mathrm{C}$. After rinsing with $0.1 \mathrm{M} \mathrm{PBS}(120 \mathrm{~mm} \mathrm{NaCl}$ in $0.1 \mathrm{M}$ phosphate buffer, $\mathrm{pH}$ 7.2), the animals were washed twice in $1 \%$ Triton X-100 in PBS for $15 \mathrm{~min}$, incubated in a 1:300 dilution of extravidin-peroxidase conjugate (Sigma) in PBS containing 0.5\% Triton X-100 for $2-3 \mathrm{hr}$, washed again in PBS, presoaked in $0.08 \%$ diaminobenzidine (DAB) in PBS for $5 \mathrm{~min}$, moved to $0.075 \%$ hydrogen peroxide in DAB solution for
A

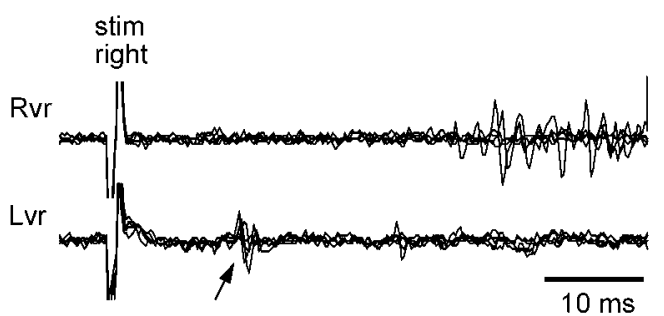

B

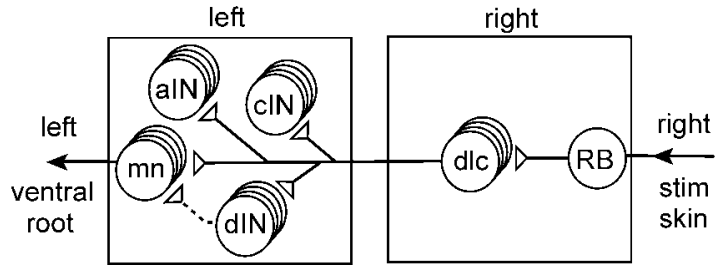

Figure 1. The spinal flexion reflex. $A$, Recordings from left (Lvr) and right (Rvr) midtrunk ventral roots to show short-latency activity (arrow) in left-side motoneurons after a $1 \mathrm{msec}$ current pulse to the right tail skin (at artifact: stim right). Five traces overlapped. B, Spinal cord neurons that may be parts of the response pathway from skin touch on the right side to motoneuron activity on the left. The direct pathway leads from skin touch-sensory RB neurons to sensory pathway dlc interneurons, across the ventral commissure to motoneurons on the left side. Other possible targets for dlc interneuron excitation are central pattern generator interneurons active during swimming: alN that produce glycinergic inhibitory gating of dlc interneurons during swimming (Li et al., 2002), glycinergic reciprocal inhibitory cIN, and dIN that are thought to produce glutamatergic excitation during swimming. Open circles represent populations of interneurons; small open triangles are presumed glutamatergic synapses.

$5 \mathrm{~min}$, and then washed in running tap water. The brain and spinal cord were then dissected free with the notochord and some ventral muscles, dehydrated, cleared in methyl benzoate and xylene, and whole-mounted between two coverslips using Depex (BDH Chemicals, Poole, UK).

Neurons were observed using a $100 \times$ oil immersion lens and traced using a drawing tube. To compensate for shrinkage during dehydration, all measurements in this study have been corrected by multiplying by 1.28 (Li et al., 2001).

\section{Results}

\section{Short-latency flexion reflex to tail-skin stimulation}

By making recordings from the motor nerves (ventral roots) in the midtrunk swimming muscles on both sides of the tadpole's body, we defined the normal response of spinal motoneurons to tail-skin stimulation. To allow us to measure latencies accurately, we used a 1 msec current pulse to excite the peripheral neurites of primary touch-sensitive RB neurons that innervate the skin (Clarke et al., 1984). Typically, the first motoneuron activity is a brief, short-latency, ventral-root burst on the opposite side (Fig. $1 A)$. This is then followed by the onset of swimming. The latency of the initial contralateral response to a near-threshold stimulus was measured for eight animals and lay in the range of 7-13.8 msec $(11.5 \pm 2.7 \mathrm{msec})$.

\section{Connections from skin-sensory neurons to sensory pathway interneurons}

In Xenopus tadpoles, we have detailed anatomical definitions for spinal-sensory neurons, motoneurons, and interneuron classes (Clarke and Roberts, 1984; Roberts et al., 1999; Li et al., 2001). We also know about the responses of most of these neurons to skin stimulation, their activity during fictive swimming, and details about some of their synaptic connections (Dale, 1985; Dale and Roberts, 1985; Li et al., 2002). We therefore knew the candidate neurons in the pathway from touching the skin on one side to 

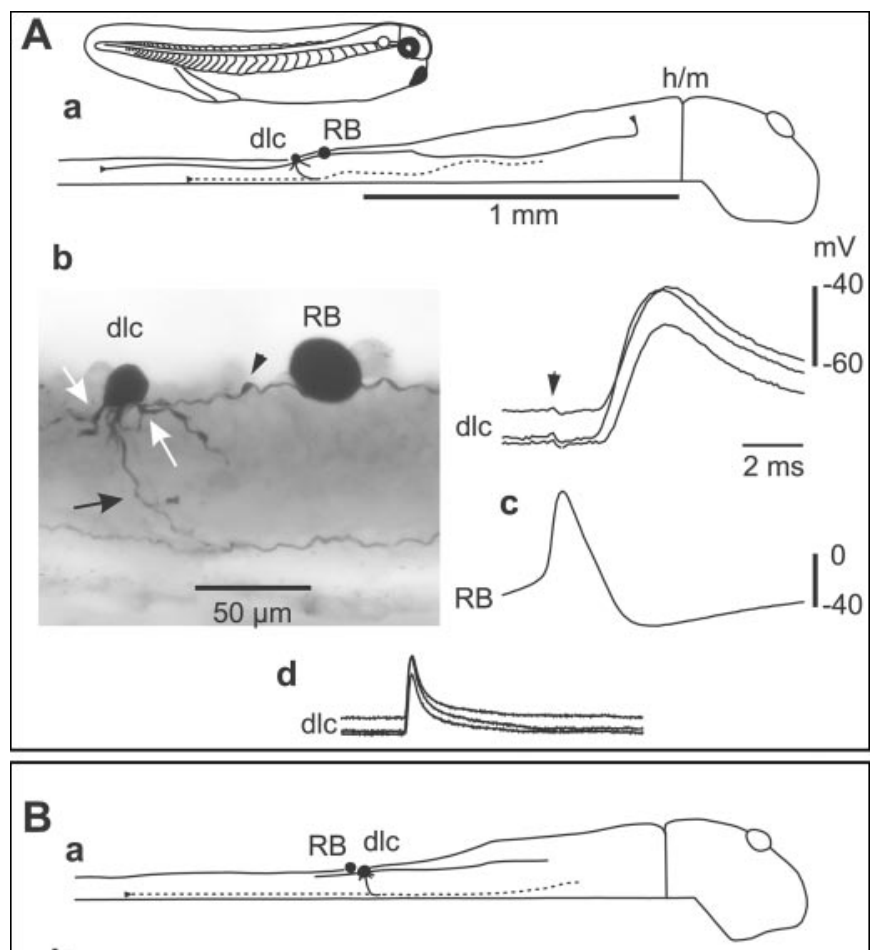

b
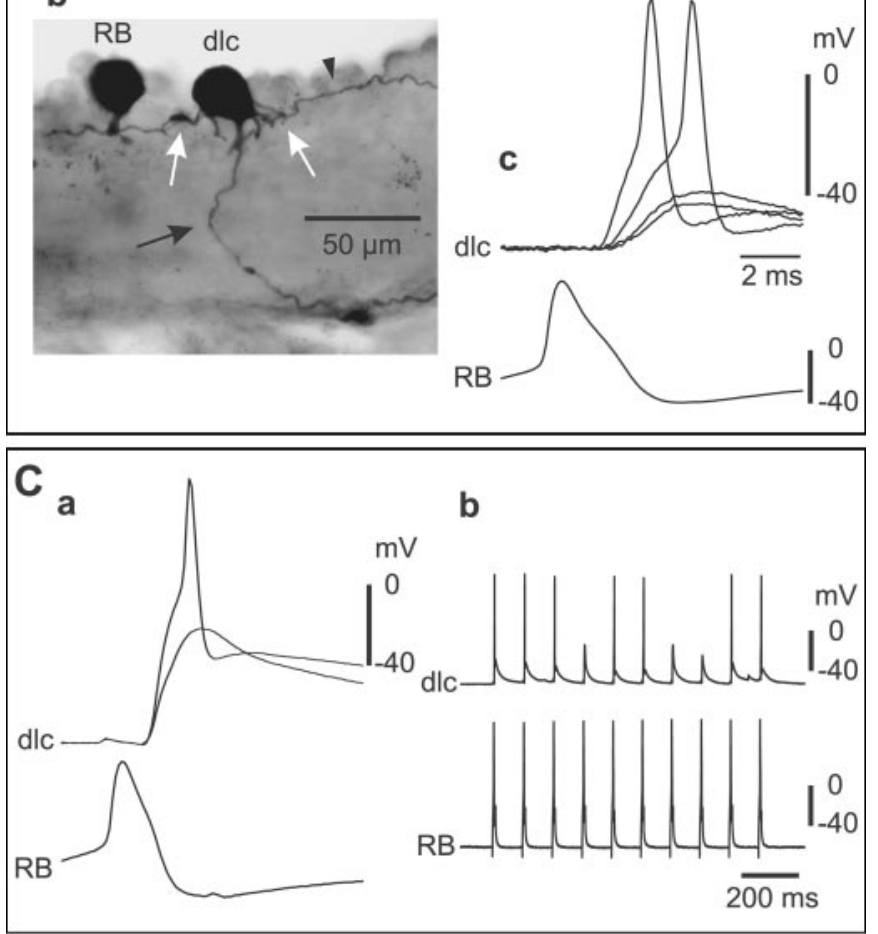

Figure 2. Paired recordings show that $\mathrm{RB}$ sensory neurons strongly excite sensory pathway dlc interneurons at a short and constant latency. $A, a$, Tadpole 5- $6 \mathrm{~mm}$ long and a scale diagram of the CNS show a filled RB and dlc interneuron. The dlc axon (dotted line) is on the opposite left side; the hindbrain to midbrain border is indicated $(\mathrm{h} / \mathrm{m}) . b, \mathrm{RB}$ and dlc interneuron at the cut dorsal edge of the spinal cord in the fifth segment on the right side. The spherical RB soma has an ascending axon and descending axon (arrowhead) with possible contact sites onto dlc dendrites (white arrows). The multipolar dlc soma has oblique dendrites and a ventral initial process leading to a ventral commissural axon (black arrow). c, Current injection evoking an action potential in RB leads to a large EPSP in the dlc interneuron at short latency (three traces overlapped; the whole EPSP, trace length, $100 \mathrm{msec}$, is shown in $d$ ). The RB impulse causes a small artifact in the dlc interneuron (arrowhead). $B, a$, In this case, the RB is caudal to the dlc interneuron. b, Ascending RB axon (arrowhead) with possible contacts to the dlc interneuron (white arrows) that has a commissural axon (black arrow). c, Current-evoked RB activity in motoneurons on the other (Fig. $1 B$ ). We first examined connections from sensory neurons (RB) to sensory interneurons (dlc interneurons) in the dorsolateral region of the cord.

With the spinal cord opened along its dorsal midline, RB neurons can be recognized by their large size and position at the cut surface. We made simultaneous recordings from $\mathrm{RB}$ neurons and 48 other neurons with somata exposed by the dorsal cut using a 0 $\mathrm{Mg}^{2+}$ saline so the NMDAR-mediated component of glutamatergic excitation would be clear. Possible RB neurons showed no response or a single spike to skin stimulation and no input during fictive swimming (Clarke et al., 1984). Possible dlc interneurons showed a short-latency EPSP to skin stimulation that often led to an action potential and were inhibited during fictive swimming (Clarke and Roberts, 1984; Roberts and Sillar, 1990). In 28 of these paired recordings, intracellular current injection that evoked an action potential in the RB neuron led to a large EPSP in the other neuron (Fig. 2). In 15 cases, the EPSP was sufficient to evoke an action potential from the resting membrane potential. Later anatomical processing confirmed the identification of RB neurons by their spherical soma, absence of dendrites, and ascending and descending central axons in the dorsal spinal cord (Clarke et al., 1984). The anatomy also showed that each postsynaptic neuron had the following features of dlc interneurons: a dorsolateral multipolar soma with a number of dendrites, and a ventral initial process leading to a ventral commissural axon that projects toward the brain on the opposite side but also branches to project at least a short distance toward the tail (Fig. 2A) (Roberts and Sillar, 1990; Li et al., 2001).

EPSPs were examined for 15 neuron pairs in which the anatomy of presynaptic RB neurons and postsynaptic dlc interneurons was clear. Median EPSP amplitude for each pair ranged from 3.4-25.4 $\mathrm{mV}$, and maximum amplitudes ranged from 6.5-33.5 $\mathrm{mV}$. EPSP amplitude did not correlate with the dlc interneuron resting potential $(-61.7 \pm 5.8 \mathrm{mV})$. All EPSPs had a fast rise in the range from 1.1-2.9 msec (overall median, $1.6 \mathrm{msec}$ ). For 14 pairs, EPSP duration at 50\% peak amplitude ranged from 5.5$22.9 \mathrm{msec}(12.6 \pm 5.9 \mathrm{mV})$. For one pair, the duration was much longer (median, $132.3 \mathrm{msec}$ ), suggestive of a polysynaptic component. The fast rise and relatively short duration suggested that the other EPSPs were primarily produced by activation of non-NMDARs.

If $\mathrm{RB}$ axons make direct monosynaptic connections onto dlc interneurons, then EPSP latencies should be short and consistent. All EPSP latencies were short, ranging from $1.4-3.4 \mathrm{msec}$ ( $2.34 \pm$ $0.55 \mathrm{msec} ; n=15$ neuron pairs; measured from the peak of the presynaptic spike to $10 \%$ of the peak amplitude on the EPSP). Consistent latency was shown by a low SD in latency values for each pair (range, $0.05-0.51 \mathrm{msec}$ ). The distances between the RB and dlc interneuron somata were $0.09-0.61 \mathrm{~mm}$ when measured anatomically from the neurobiotin fills. For each pair, the minimum EPSP latency increased significantly with conduction distance $(p=0.027)$. Assuming a conduction velocity for the $\mathrm{RB}$ axons of $0.3 \mathrm{~m} / \mathrm{sec}$ [the modal value quoted by Clarke et al. (1984)], we estimated the mean synaptic delay to be $\sim 1 \mathrm{msec}$.

action potentials lead to variable amplitude dlc interneuron EPSPs, two of which reach a threshold for a dlc action potential. C, a, An example in which current-evoked action potentials in an RB in the sixth segment lead to large, reliable, constant-latency EPSPs in a dlc interneuron. Even with -30 pA hyperpolarizing current injected into the dlc interneuron, these EPSPs can reach threshold and evoke dlc interneuron action potentials (time scale as in $B$ ). The reliability of the EPSP is shown by repeated current pulses to the RB neuron. 


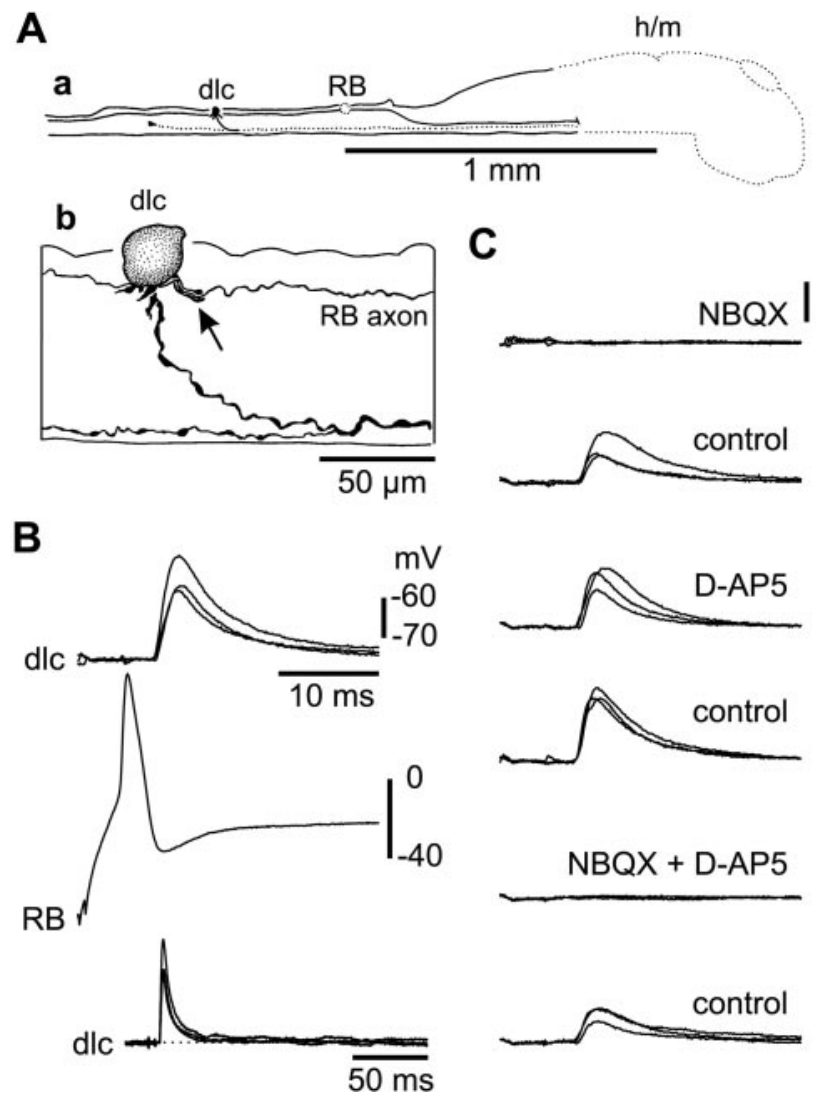

Figure 3. Effects of glutamate receptor antagonists on EPSPs evoked in a dlc interneuron by $\mathrm{RB}$ action potentials. A, Scale drawings of the CNS from the right side to show the location of the RB soma and its descending axon passing the dlc interneuron soma (axon dotted) ( $a$ ); at higher magnification, a possible contact point (arrow) of the RB axon and a dendrite emerging from the $\mathrm{dl}$ interneuron soma is shown $(b)$. The ventral, commissural dlc interneuron axon branches on the opposite side of the cord are shown. $B$, Top two traces, Current evoked-RB impulses (RB) produce large EPSPs in the dlc interneuron (dlc). Bottom trace, Whole time course of dlc interneuron EPSPS. C, The EPSP is blocked by bath application of NBQX, partially recovers in control, and is little affected by D-AP-5. After recovery in control, the EPSP is blocked by simultaneous application of $2.5 \mu \mathrm{m}$ NBQX and $25 \mu \mathrm{M}$ D-AP-5 and recovers in control. Antagonists were dropapplied (three overlapped traces in each).

This is fully compatible with the connection between RB axons and dlc interneurons being monosynaptic.

To investigate the receptors that were activated when RB action potentials led to excitation of dlc interneurons, we looked at the effects of the specific NMDAR antagonist D-AP-5 and AMPAR antagonist NBQX, as well as $1 \mathrm{mM} \mathrm{Mg}^{2+}$, which would be expected to block any voltage-dependent NMDAR-mediated component. When the antagonists were applied during paired recordings, EPSPs evoked in dlc interneurons by $\mathrm{RB}$ impulses appeared to be completely blocked by $5 \mu \mathrm{M}$ NBQX $(n=12)$ but were little affected by D-AP-5 $(n=8)$ (Fig. 3$)$ or $1 \mathrm{mM} \mathrm{Mg}^{2+}(n=$ 4; data not shown).

To determine whether excitation of anatomically identified dlc interneurons was primarily AMPAR-mediated, as suggested by the short time course, we recorded dlc interneuron responses to electrical stimulation of RB processes in the ipsilateral tail skin (Fig. 4). Under both current clamp $(n=10)$ and voltage clamp $(n=9)$, these recordings showed that the fast early component of excitation, blocked by NBQX, provided the majority of the peak potential or current (see below). The slower, longer component blocked by D-AP-5 and $1 \mathrm{mM} \mathrm{Mg}^{2+}$ was much smaller. The AMPAR and NMDAR currents were separated pharmacologi- cally by the addition of D-AP-5 or NBQX, respectively (Fig. 4C). The peak current was then measured under voltage clamp (at $-65 \mathrm{mV}$ ) for nine dlc interneurons. The peak AMPAR current was $\sim 2$ msec after EPSC onset and ranged from 101-444 pA. The peak NMDAR current was $\sim 20-30 \mathrm{msec}$ after EPSC onset and ranged from 3-36 pA. On the basis of these peak currents, the AMPAR/NMDAR ratio for the nine neurons was $28 \pm 31.7$ (range, 6.5-108). The synapses from RB to dlc interneurons are therefore dominated by AMPAR-mediated excitation. We conclude from these results that sensory RB neurons synapse directly with sensory pathway dlc interneurons and release glutamate that activates primarily AMPAR to produce strong excitation, in which individual EPSPs can often lead to dlc interneuron action potentials.

\section{Connections from dlc interneurons to contralateral neurons}

To investigate the synaptic connections from dlc interneurons, we made 58 simultaneous recordings from dlc interneurons on the right side of the spinal cord and neurons on the contralateral left side. In many cases, the medial surfaces of the somata of neurons on the left side were exposed in more ventral regions by making small cuts in the wall of the central canal. All of these neurons were identified by examining their anatomical features after the experiment. All showed rhythmic activity during fictive swimming (not illustrated), and their responses to right-side (contralateral) skin stimulation were recorded.

Thirteen of the left-side neurons were identified as motoneurons (Fig. 5A). Because muscles had to be removed at an early stage in the dissection, peripheral axons that would give unambiguous identification of motoneurons were often lost. In these cases, we therefore adopted the following criteria for identification [on the basis of Roberts et al. (1999)]: multipolar soma, short ventral descending axon ( $<100 \mu \mathrm{m}$ in length), possible broken axon end at the surface of the spinal cord, no broken axon end near the ventral midline. When current evoked an action potential in the right-side dlc interneuron, six motoneurons received small, long-duration, short-latency EPSPs (Fig. $5 B, C$ ). The remaining 45 left-side neurons were premotor interneurons belonging to three anatomical classes. Commissural interneurons (cINs; $n=36$ ) are glycinergic and produce reciprocal inhibition during both swimming and struggling (Dale, 1985; Soffe, 1993). These cINs have unipolar somata, an initial ventral process with short dendrites, and a ventral commissural axon that branches to ascend and descend contralaterally (Fig. 5D) (Yoshida et al., 1998). In 18 cases, dlc interneurons produced small, longduration, short-latency EPSPs in cINs (Fig. $5 E, F$ ).

Six ascending interneurons (aINs) were recorded with dlc interneurons, and in four cases there were synaptic connections. These glycinergic interneurons have unipolar somata lying in the dorsal half of the cord with primarily ventral dendrites (Fig. 6A). They have an ascending axon that branches near the soma to give a descending axon, are active during swimming, and produce gating inhibition of sensory pathway dlc interneurons (Li et al., 2002). Impulses in dlc interneurons led to small, long-duration, short latency EPSPs in all four aINs. Three examples of descending interneurons (dINs) were recorded with dlc interneurons. These are thought to be the excitatory glutamatergic interneurons responsible for excitatory drive during swimming (Dale and Roberts, 1985). They have multipolar somata and a descending ipsilateral axon (Li et al., 2001) (Fig. 7A). In one paired recording, dlc interneuron impulses led to a small EPSP in a dIN (not illustrated).

The EPSPs evoked by dlc interneuron impulses in contralat- 
A

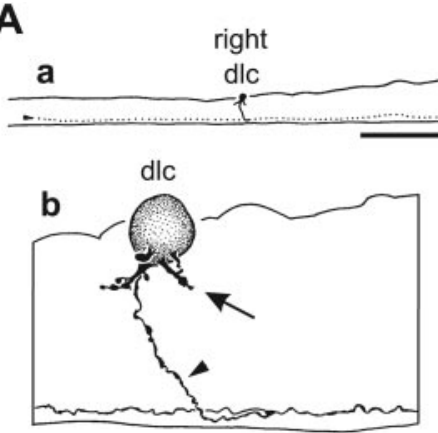

B

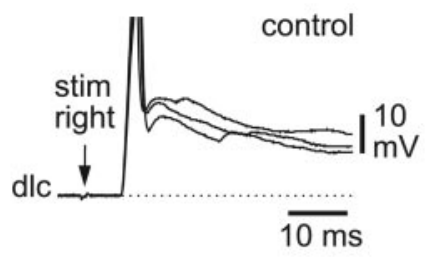

NBQX

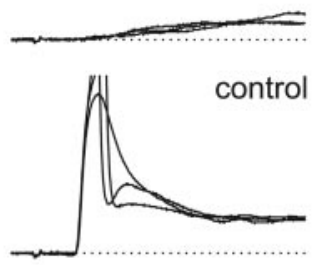

D-AP5

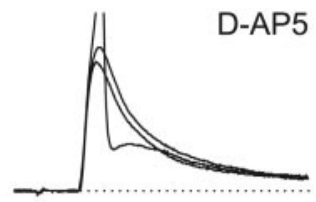

control

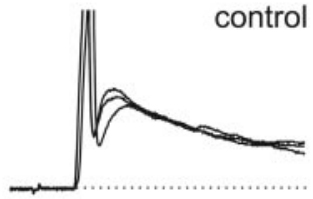

NBQX + D-AP5

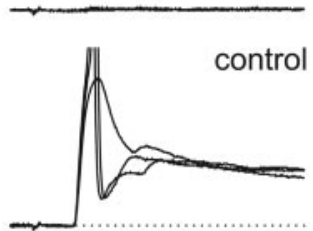

C stim right

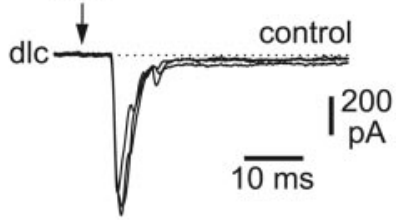

NBQX

control

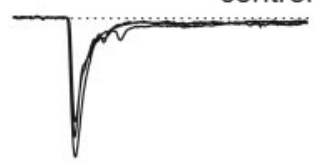

D-AP5

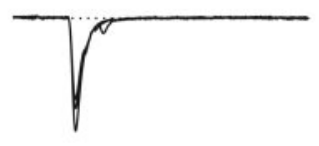

control

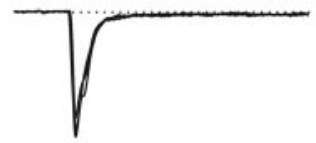

$\mathrm{NBQX}+\mathrm{D}-\mathrm{AP5}$

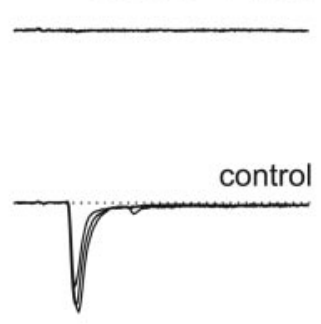

Figure 4. Effects of glutamate receptor antagonists on excitation in dlc interneurons produced by electrical stimulation of RB neurites in the skin. $A$, CNS from the rightside to show the location of the dlc soma and contralateral axon ( $a$, dotted) and the characteristic dlc soma with emerging dendrites ( $b$, arrow) and ventral axon ( $b$, arrowhead). $B$, Current-clamp recordings in which the RB stimulus (arrow) produces an EPSP and action potential in control. The EPSP is strongly reduced in NBQX, little affected by D-AP-5, and blocked by combined application. C, Another dlc interneuron, voltage-clamped at $-65 \mathrm{mV}$, shows a large, fast, inward current in response to the RB stimulus (arrow). The large fast component is blocked by $2.5 \mu \mathrm{m} \mathrm{NBQX}$ to leave a small slow component that is blocked by $25 \mu \mathrm{m}$ D-AP-5. Antagonists were drop-applied (three overlapped traces in each case). Strychnine ( $2 \mu \mathrm{M})$ was in the saline to block glycinergic inhibition on the dlc interneuron.
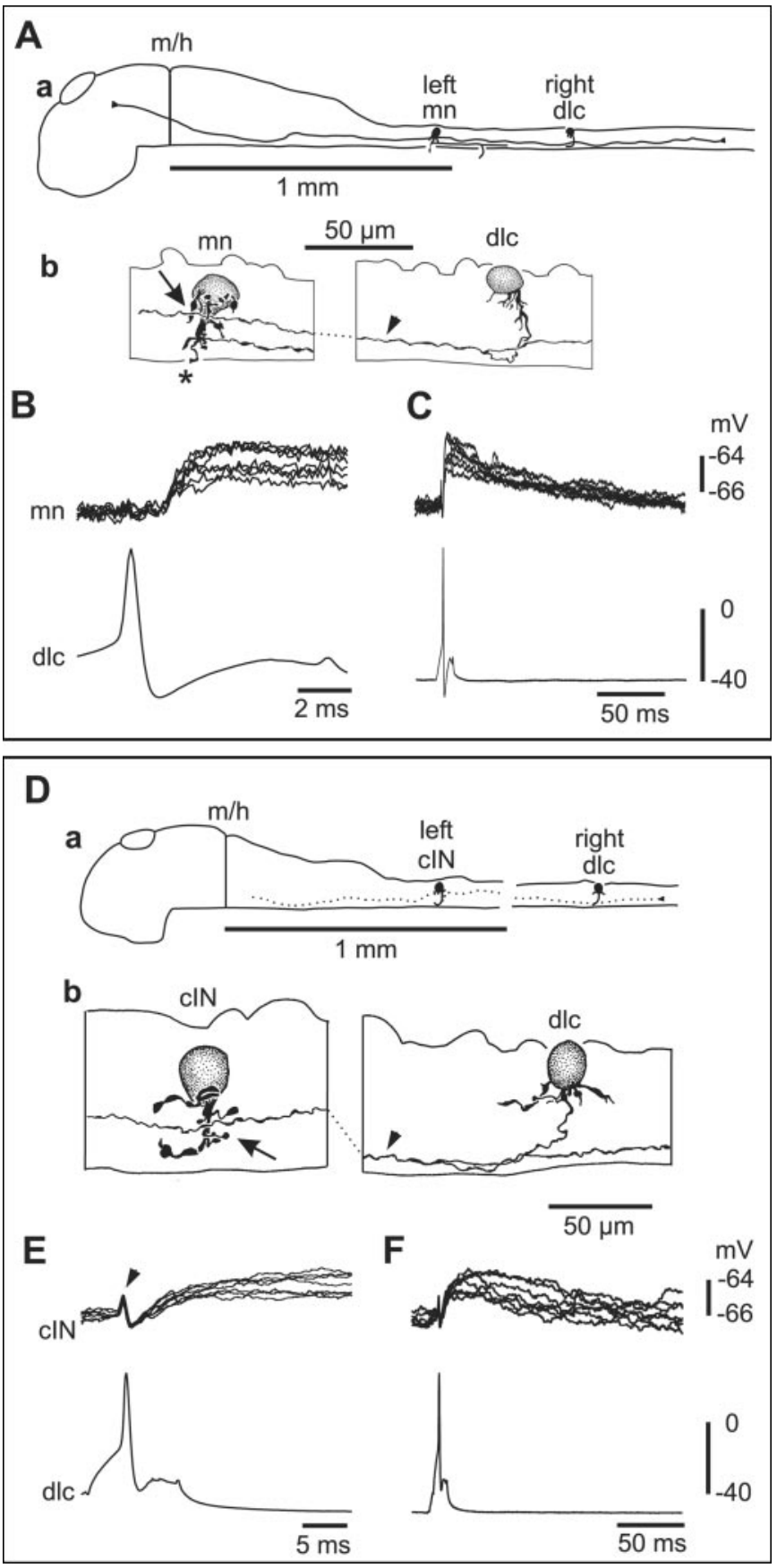

Figure 5. Paired recordings show that sensory pathway dlc interneurons excite a contralateral motoneuron and commissural IN at short and constant latency. $A, a$, Scale drawing of the CNS viewed from the left side to show the dlc interneuron, with multipolar soma at the dorsal edge of the spinal cord on the right side, has a ventral commissural axon that branches after crossing to the left side. Here, its ascending axon has possible contact sites onto a motoneuron. $b$, Multipolar motoneuron soma at the fourth segment on the left side with a possible contact point from the dlc axon (arrowhead) on its dendrites (arrow). The mn has a ventral descending axon and a broken peripheral axon (asterisk). B, Current injection evoking an action potential in the dlc interneuron, shown in $A$, leads to a small EPSP in the $\mathrm{mn}$ at constant and short latency (overlapped traces). C, The same record as $B$ showing the long duration of the EPSP.D, $a$, Scale drawing to show a right dlc interneuron and left cIN. $b$, Possible synaptic contact (arrow) between the dlc axon (arrowhead) and cIN dendrite. E, F, Small slow-rise cIN EPSPs produced by dlc spikes evoked by current injection. The artifact (arrowhead) caused by the presynaptic dlc action potential has not been subtracted. 


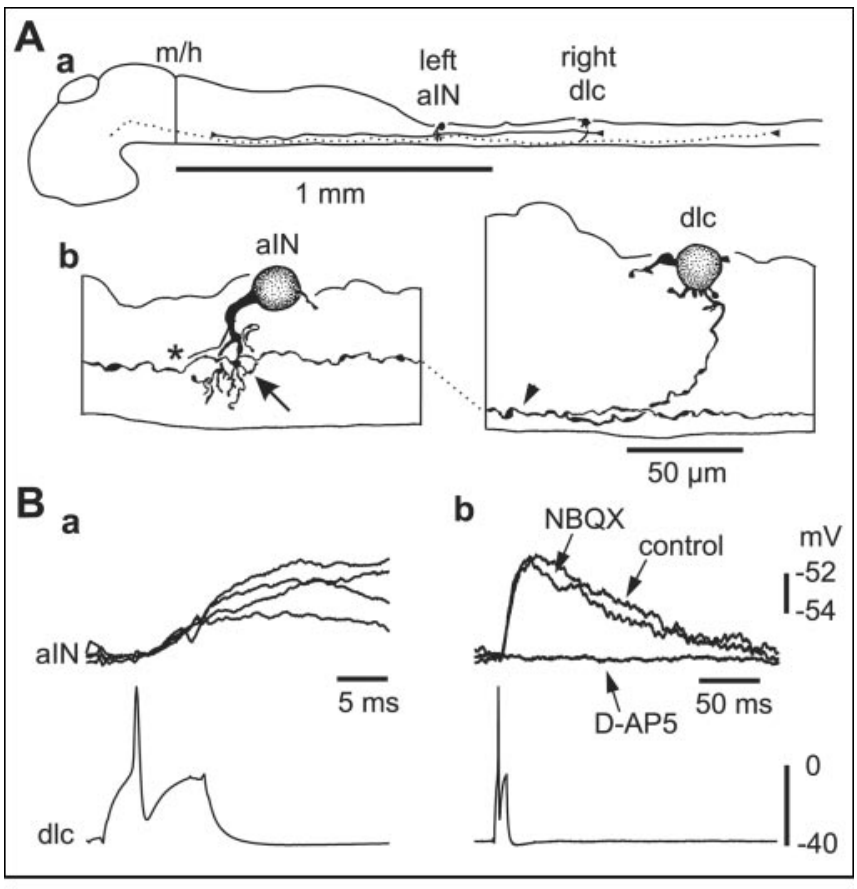

C

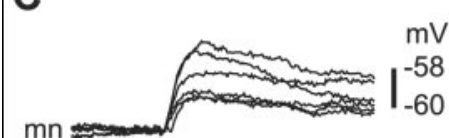

E
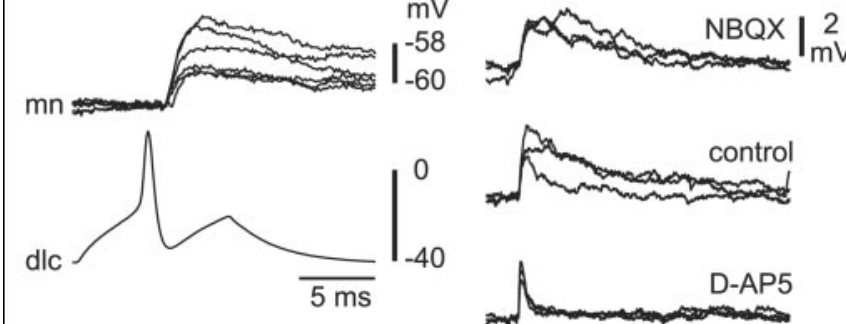

D

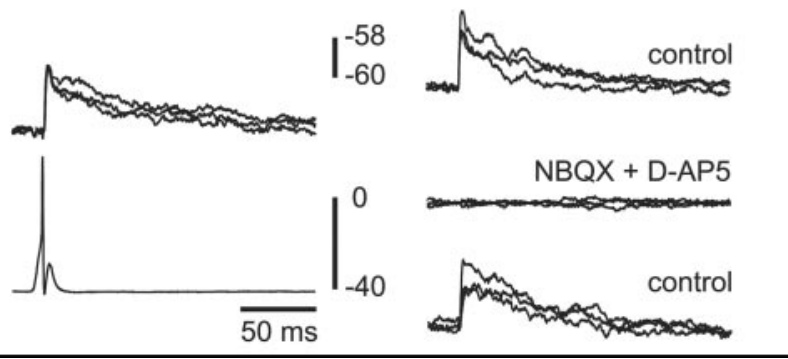

Figure 6. Paired recordings show that sensory pathway dlc interneurons excite contralateral neurons by activating glutamate receptors. $A, a$, Scale drawings of the CNS viewed from the left side to show a dlc interneuron on the right side of the spinal cord with a commissural axon that branches on the left side, where its ascending axon (dotted) has possible contact sites onto an alN. $b$, The multipolar dlc interneuron soma has an ascending axon (arrowhead) with possible contact points (arrow) on dendrites of the alN, the axon (asterisk) of which has been omitted for clarity. $B, a$, Current injection evoking an action potential in the dlc interneuron shown in $A$ leads to slow-rise, small EPSPs in the alN at short latency (four overlapped traces). $b$, The same neuron pair shows that the long-duration EPSP is blocked by $50 \mu \mathrm{m} \mathrm{D}-A \mathrm{P}-5$ but is little affected by $5 \mu \mathrm{m}$ NBQX.C, Small, short-latency EPSPs (six overlapped traces) in a mn produced by current-evoked $\mathrm{dlc}$ interneuron impulses (dlc). D, The same recording as ( showing the long duration of the EPSP. E, Effects of glutamate receptor antagonists on EPSPs evoked in the motoneuron by dlc interneuron action potentials. The long, slow component of the EPSP is blocked by $50 \mu \mathrm{m}$ D-AP- 5 and recovers in wash. The fast initial component is blocked by $5 \mu \mathrm{m} \mathrm{NBQX}$. After recovery in wash, the EPSP is blocked by simultaneous application of $5 \mu \mathrm{MNBQX}$ and $50 \mu \mathrm{m} \mathrm{D}-\mathrm{AP}-5$ and returns after wash. Antagonists were drop-applied (three overlapped traces; time scale as in D).

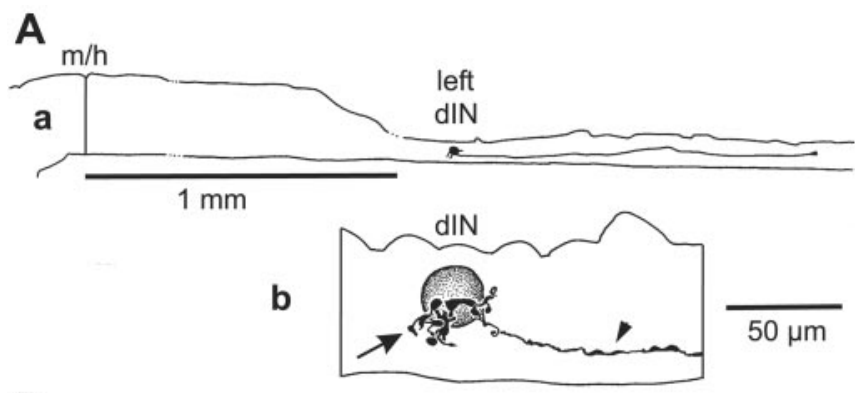

B
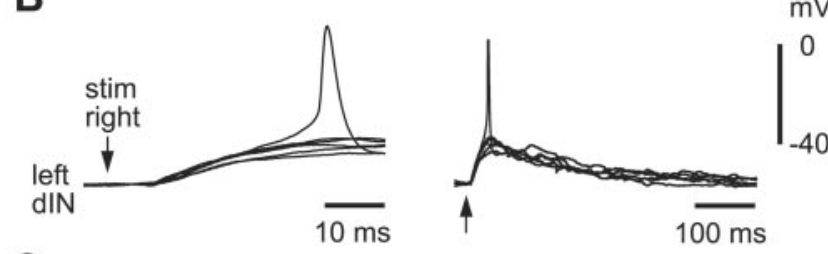

C stim
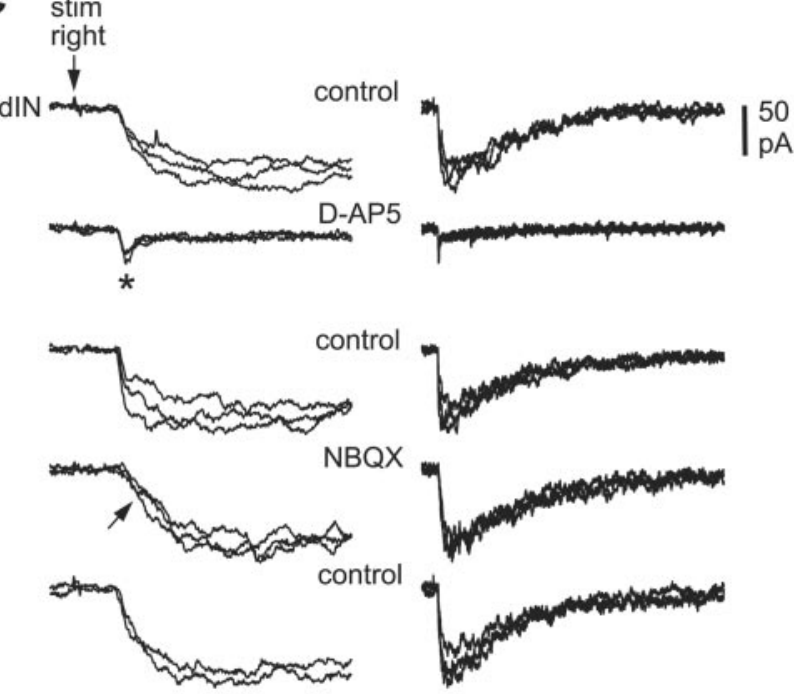

Figure 7. Pharmacology of responses to contralateral skin stimulation in a dIN. $A, a$, Scale drawing of the CNS viewed from the left side to show the dIN on the left side of the spinal cord with a descending axon. $b$, Details of the dIN multipolar soma with short dendrites (arrow) and a descending axon (arrowhead). B, Contralateral tail-skin stimulation (arrow) produces a slowrise EPSP at constant latency that, in one trace, evokes a spike in the dIN. C, Pharmacology of EPSCs in the same dIN at a holding potential of $-65 \mathrm{mV}$. D-AP- 5 blocks a slow component to reveal a small fast EPSC (asterisk), which is blocked by NBQX (arrow). Antagonists were microperfused, and the circulating saline contained $10 \mu \mathrm{m}$ mecamylamine and $2 \mu \mathrm{m}$ strychnine to block any cholinergic and glycinergic currents (three overlapped traces; time scales as in $B$ ).

eral neurons (motoneurons and interneurons) differed from RBevoked EPSPs in dlc interneurons in three ways. First, they had significantly lower peak amplitudes (combined measurements from 22 neurons, $2.9 \pm 2.5 \mathrm{mV}$; range, 0.6-11.8 mV; MannWhitney; $p<0.001)$. Second, the rise times of EPSPs varied widely but were of broadly two types. Some $(\sim 70 \%)$ were relatively slow, ranging from $10-40 \mathrm{msec}(n=15)$ (Figs. $5 E, 6 B)$; the remainder $(\sim 30 \%)$ were clearly fast $(<5 \mathrm{msec} ; n=6)$ (Figs. $5 B$, $6 C$ ). Third, the EPSPs had a significantly longer duration (combined measurements from 20 neurons at half-peak amplitude, $68.4 \pm 49.4 \mathrm{msec}$; range, $14-236 \mathrm{msec}$; Mann-Whitney; $p<$ 0.001) (Fig. 5C,F).

In some of these motoneuron and premotor IN recordings, the small size and short latency of the EPSP meant that its onset could not be clearly distinguished from the coupling artifact produced by the presynaptic impulse (Figs. $5 E, 6 B$ ). However, the 
onset was clear in averaged records from the remaining 18 examples after subtraction of the artifact (see Materials and Methods) (Figs. 5A, 6C). Latencies in these examples (with distances between presynaptic and postsynaptic neurons from $0.2-0.7 \mathrm{~mm}$ ) ranged from $0.8-3.1 \mathrm{msec}$, and the mean value with its low SD $(1.72 \pm 0.62 \mathrm{msec})$ was similar to that for RB to dlc interneuron synapses. Assuming the same conduction velocity for the dlc axons as used for RB axons above $(0.3 \mathrm{~m} / \mathrm{sec})$ (Clarke et al., 1984), we estimated the mean synaptic delay to be $\sim 0.2 \mathrm{msec}$. This rather low value suggests that the conduction velocity for dlc axons may actually be faster than that for RB axons but is still fully compatible with dlc interneurons making direct monosynaptic connections to contralateral neurons. We conclude that motoneurons and the three known premotor interneuron classes that are active during swimming (aINs, dINs, and cINs) all receive direct weak excitation from contralateral dlc interneurons.

\section{Pharmacological properties of synapses in contralateral neurons}

The long duration of EPSPs evoked in contralateral neurons by dlc interneuron impulses, together with the long rise-time of some EPSPs, suggested the presence of a significant NMDARmediated component. Application of D-AP-5 and NBQX to EPSPs evoked by spikes in a single dlc interneuron $(n=7$ neuron pairs) (Fig. $6 B, E$ ) or by a current pulse to the contralateral tail skin ( $n=8$ neurons; not illustrated) showed that most of these EPSPs had both an AMPAR and NMDAR component (Fig. 6). The NMDAR component of the EPSP was relatively larger than that for EPSPs in dlc interneurons. Similar results were obtained for nine additional neurons under voltage clamp (one motoneuron, eight interneurons), in which EPSCs were evoked by a current pulse to the contralateral tail skin (Figs. $7 C, 8 A--C$ ). The long NMDAR component of the dlc interneuron-evoked EPSC was also blocked by adding $1 \mathrm{mM} \mathrm{Mg}^{2+}$ to the bathing saline $(n=6)$ (Fig. 8D).

In contrast to the strong AMPAR component that dominated the excitation in dlc interneurons, the AMPAR component of dlc interneuron output synapses was especially variable. In some paired records, the AMPAR component appeared to be absent (Fig. $6 \mathrm{Bb}$ ), but it was larger in others. Under voltage clamp, some EPSCs had a significant AMPAR component and showed a fast rise ( $<5$ msec to peak) (Figs. $5 C, 6 C$ ), whereas others had little or no AMPAR component and showed a much slower rise $(\sim 20$ msec or more to peak) (Figs. $5 F, 6 B, 7 B$ ).

The relative contributions of AMPAR and NMDAR currents were measured under voltage clamp for EPSCs evoked by stimulation of the contralateral tail skin in nine neurons (one motoneuron and eight interneurons) (Figs. 7C, $8 B--D$ ). For EPSCs in dlc interneurons, the peak AMPAR current was $\sim 2$ msec after EPSC onset but was smaller and ranged from only 2-108 pA. Peak NMDAR current was $\sim 20-30$ msec after EPSC onset and ranged from 6-84 pA. The AMPAR/NMDAR ratio on the basis of these peak currents was $1.51 \pm 1.43$ (range, $0.23-4.64$ ). A very similar ratio of $1.31 \pm 0.62$ (range, $0.30-2.05$ ) was obtained in six additional motoneurons by comparing the amplitudes of EPSCs at 2 and $20 \mathrm{msec}$ from the onset, which is equivalent to the times of peak AMPAR and NMDAR currents revealed pharmacologically. The AMPAR/NMDAR ratio for motoneuron and IN EPSCs was significantly lower than the ratio of $28.0 \pm 31.7$ for EPSCs in dlc interneurons (Mann-Whitney; $p<0.001$ ). Unlike the EPSCs evoked by RBs in dlc interneurons, the EPSCs, which dlc interneurons evoke in neurons on the opposite side of the spinal cord, have a significantly stronger NMDAR-mediated component.
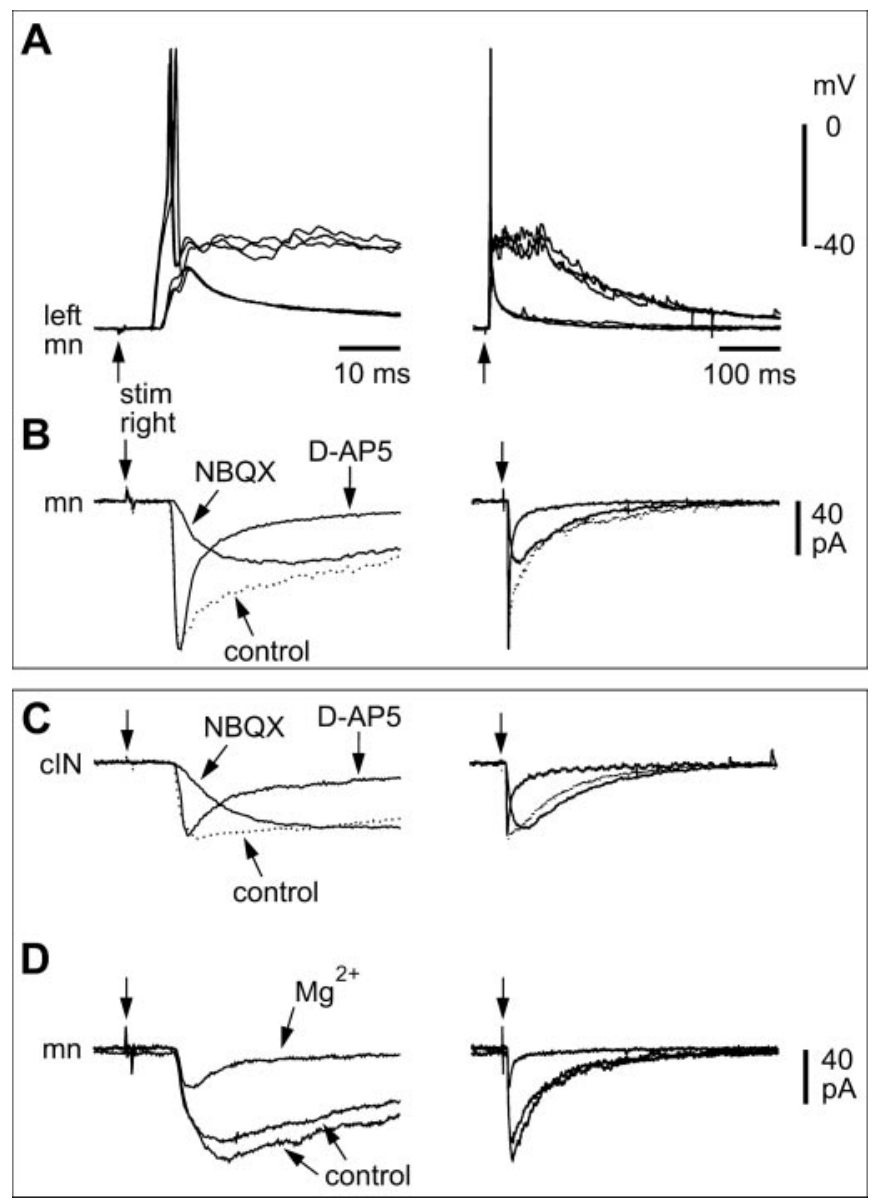

Figure 8. Pharmacology of excitation evoked by contralateral skin stimulation (arrows) and effects of $\mathrm{Mg}^{2+}$. For each example, the inital (left) and whole (right) response is shown. $A$, EPSPs in a motoneuron that evoke an action potential at higher stimulus levels (overlapped traces). $B$, In the same $\mathrm{mn}$, averaged EPSCs in control saline, NBQX and D-AP-5, as used in measurements of AMPAR/NMDAR current ratios. C, Similar averaged EPSCs from a cIN. D, Effect of $1 \mathrm{mM} \mathrm{Mg}^{2+}$ on averaged slow-rise EPSCs in another mn. Antagonists and $\mathrm{Mg}^{2+}$ were microperfused close to the recorded neuron, and the circulating saline contained $10 \mu \mathrm{m}$ mecamylamine and $2 \mu \mathrm{m}$ strychnine.

\section{Role of sensory pathway dlc interneurons}

Our evidence suggests that dlc interneurons produce two types of excitation of contralateral neurons. Some neurons, particularly motoneurons, have a clear AMPAR component to their excitation. In response to skin stimulation, this component can summate to produce firing from the resting state (Figs. $8 A, 9 A$ ). Other neurons receive weaker, primarily NMDAR-mediated excitation that is very unlikely to lead to firing from rest in normal saline with $\mathrm{Mg}^{2+}$ (Fig. 6). What is the function of these different types of excitation of contralateral neurons?

The faster AMPAR-mediated excitation could produce the first short-latency contralateral motor response seen when swimming starts in response to skin stimulation (Fig. 1A). A typical pattern of sensory pathway interneuron and motoneuron activity underlying this type of reflex action is shown in Figure 9A. Similar short-latency $(<15 \mathrm{msec})$ action potentials in response to skin stimulation were found in $28 \%$ of recordings from contralateral motoneurons (7 of 25) (Fig. 8A) and 12\% of those from contralateral interneurons (5 of 41).

A second role for AMPAR excitation in neurons excited by dlc interneurons is to produce "extra" contralateral motoneuron impulses at short latency when skin stimulation occurs during on- 

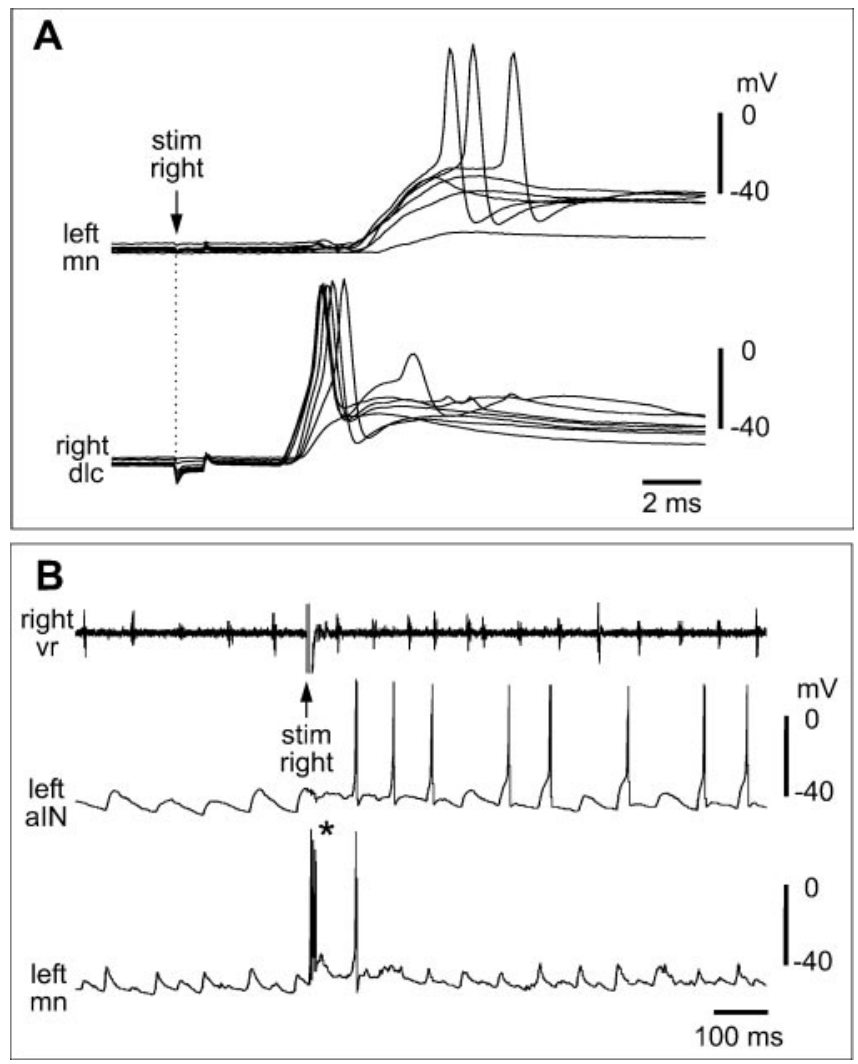

Figure 9. Roles of excitation from dlc interneurons. $A$, Excitation of a contralateral $\mathrm{mn}$ from rest. A paired recording from a right-side sensory pathway dlc interneuron and a left-side motoneuron is shown (the same recording as Figure $5 A$ ). Responses to increasing the strength of a current pulse to stimulate the right tail skin (arrow) are shown. At higher stimulus levels, both $\mathrm{dlc}$ and $\mathrm{mn}$ fire an action potential at latencies of $\sim 5$ and $\sim 10 \mathrm{msec}$. $B$, Excitation during swimming. When the right tail skin is stimulated (arrow), swimming frequency increases in the right-side ventral root record at the tenth segment (right, vr), and central pattern generator neurons on the left side (left alN; left $\mathrm{mn}$ ) are recruited to fire action potentials. The $\mathrm{mn}$ even fires three action potentials (*).

going swimming (Sillar and Roberts, 1988b). These extra action potentials could lead to turning responses. Such responses had only previously been studied in $0 \mathrm{Mg}^{2+}$ saline. We therefore re-examined the effects of tail-skin stimulation on contralateral neurons at different phases of the fictive swimming cycle in saline with $1 \mathrm{~mm} \mathrm{Mg}^{2+}$. As in $0 \mathrm{Mg}^{2+}$ saline, we found that stimulation could evoke extra action potentials, recruit silent neurons $(n=$ 11; 6 cINs, 3 aINs, and 2 motoneurons), and produce a transient increase in swimming frequency (Fig. 9B).

\section{Discussion}

Our first aim was to determine the organization of a vertebrate spinal reflex: defining the neurons in the pathway that allows stimulation of the skin on one side of the body to excite motoneurons on the opposite side and produce a short-latency, crossedflexion response. By defining this synaptic pathway, we were then able to ask whether the properties of the synapses are uniform at this early stage in development, or whether they are tuned to their particular functions in the reflex pathway.

\section{Definition of a primitive disynaptic reflex-withdrawal pathway}

We used whole-cell patch recordings in the Xenopus tadpole spinal cord to seek synaptic connections between pairs of neurons that were identified using previously defined physiological and anatomical criteria (for review, see Roberts et al., 2000; Li et al., 2001, 2002). Evidence from 28 pairs shows that the primary skinsensory Rohon-Beard neurons that respond naturally to stroking the skin directly excite sensory pathway dlc interneurons. These interneurons have somata and dendrites located dorsolaterally in the tadpole equivalent of the spinal dorsal horn. Their axons run ventrally across the spinal cord to form a decussating projection toward the brain corresponding to the spinothalamic tract in adult vertebrates (Brodal and Brodal, 1981). The strength of the excitatory synapses from RB neurons to dlc interneurons, and the large sample size in the present recordings, gives us confidence that dlc interneurons form the pathway to carry excitation from skin touch to the other side of the spinal cord (Clarke and Roberts, 1984; Roberts and Sillar, 1990).

The output connections of sensory pathway dlc interneurons were shown using paired recordings with neurons on the opposite side, which were active during fictive swimming. In 29 cases, dlc interneurons produced direct excitation of these neurons, and in six of these cases the postsynaptic neuron was a motoneuron. This means that we have defined a minimal disynaptic pathway from cutaneous receptors on one side of the body to motoneurons on the other (Fig. $1 B$ ). Such a pathway can explain short-latency contralateral responses to electrical skin stimulation (Fig. $1 A$ ) and the normal contralateral flexion response seen in the whole behaving tadpole when the skin on one side of the trunk is stroked (Boothby and Roberts, 1995). This response is a primitive withdrawal reflex; it bends the trunk away from a stimulus and normally leads to the tadpole swimming away. Our evidence from paired recordings confirms previous proposals on the mechanism of this reflex (Sillar and Roberts, 1992).

We know of no other case in the vertebrates in which the neurons responsible for such a simple reflex have been defined. In mammals, withdrawal reflexes after skin stimulation are usually considered to be trisynaptic with two layers of interposed interneurons. However, in two cases, central delay times of $1.5 \mathrm{msec}$ or less have been calculated (Illert et al., 1976; Fleshman et al., 1988). Similar short delays of $1.6 \mathrm{msec}$ were found in jaw-opener responses to stimulation of the inferior dental nerve (Kidokoro et al., 1968). In all of these cases, the authors concluded that motoneurons must be excited via a disynaptic pathway after sensory nerve stimulation. The interneurons responsible, of course, have not been identified. It is important to remember that in the tadpole, all connections are made by fine unmyelinated axons $(<0.5$ $\mu \mathrm{m}$ in diameter). The resulting slow central delay times explain why the reflex latency may appear relatively long for a simple disynaptic pathway.

Sensory pathway dlc interneurons, as well as contralateral motoneurons, excite all classes of interneurons that are components of the central pattern generator for swimming (Fig. $1 B$ ). Descending interneurons are thought to provide the glutamatergic excitatory drive to ipsilateral motoneurons during swimming (Dale and Roberts, 1985). If they were excited to fire by contralateral skin stimulation, they would form a trisynaptic path for a withdrawal reflex that could sum with more direct input to motoneurons. If glycinergic cINs were excited to fire, they would inhibit the stimulated side to prevent conflicting action. Excitation of spinal interneurons may also play a key role in the subsequent initiation of swimming.

\section{Tuning of synaptic properties to function}

Studying the reflex pathway allowed us to see that the synapses made by primary sensory RB neurons are very different to those made by sensory pathway dlc interneurons. When recording in 0 
$\mathrm{Mg}^{2+}$ saline, the EPSPs from individual RBs to dlc interneurons were significantly larger and faster than those from individual dlc interneurons to contralateral neurons. Voltage-clamp measurements on responses to skin stimulation show that an important difference is in the size of the fast AMPAR-mediated component of conventional dual-component glutamatergic synapses. The ratio of AMPAR/NMDAR currents was 28 for RB and only 1.5 for dlc interneuron synapses. The AMPAR-mediated component dominates powerfully at RB synapses at early developmental stages. We did not measure spontaneous synaptic excitation, but EPSPs and EPSCs were recorded in TTX, in presumed dlc interneurons, and found to be large (Sillar and Roberts, 1988a; Rohrbough and Spitzer, 1999). A simple interpretation is that RB neurons may release ample transmitter at their synapses onto dlc interneurons in which the postsynaptic density of AMPAR is high. In contrast to this, synapses from dlc interneurons to contralateral neurons are weak and produce small EPSPs and EPSCs. There is only evidence for significant AMPAR-mediated excitation in some neurons. If the recordings were made in $1 \mathrm{mM} \mathrm{Mg}^{2+}$ saline, the remaining synapses would have appeared silent because they are mediated primarily by NMDARs.

What is the functional significance of these differences? The most obvious significance is that activity in individual sensory interneurons can be recruited by a few RB neurons, whereas activity in contralateral neurons will require the convergence of input from many sensory interneurons. Behaviorally, it is possible to initiate contralateral responses with a stimulus that will excite very few sensory RB neurons (1-5) by stroking a tadpole on the trunk or tail with the tip of a fine hair (Boothby and Roberts, 1995), and we can now see why. The strong synapses from RB neurons allow the recruitment of many sensory interneurons, which in turn can provide sufficient convergence of excitation to produce activity in contralateral motoneurons. The differences in these synapses could reflect different stages in the same developmental program for glutamatergic synapses. However, whether or not this is the case, it appears that the synapses are tuned to their function, as they must be in a behaving animal. The strong synapses onto sensory interneurons ensure that a response can occur to a relatively localized (possibly small) stimulus. The weaker synapses onto contralateral neurons may avoid overexcitation but will also ensure that the contralateral flexion response has a higher safety margin. Reflex responses will therefore only occur after the concerted action of many sensory interneurons that follows a significant sensory stimulus.

Our evidence suggests that the fast AMPAR component of the excitation from dlc interneurons can sum in motoneurons to produce short-latency reflex firing at rest and during swimming (Fig. 9). What is the significance of the NMDA component of dlc interneuron excitation of contralateral neurons? It is possible that the slower, weaker, voltage-dependent NMDAR-mediated excitation that would normally be blocked at rest could be "gated-in" by the excitatory synaptic drive descending from the brainstem when swimming starts. In this way, this excitation could contribute to a snowballing positive feedback to help initiate motor activity. It could also be gated-in by the excitatory synaptic drive present during swimming (cf. Reichert and Rowell, 1985). In this way, NMDAR-mediated dlc interneuron excitation could have an influence beyond the simple reflex response, producing longer lasting increases in swimming frequency, either directly or indirectly by recruitment of premotor excitatory interneurons (Sillar and Roberts, 1988b, 1992).

\section{Conclusion}

We defined the neuronal pathway for a simple withdrawal reflex in the frog tadpole. The evidence at this early stage of development shows that synapses at different steps in this pathway have very different properties that appear to be tuned to their functions in the reflex response. It is possible that these differences result from the sensory neurons and interneurons being at different stages in a common developmental program in which synapses move from NMDA- to AMPA-mediated excitation. However, the only study on the development of these sensory neuron synapses in Xenopus has suggested the opposite, a move from more AMPA to more NMDA receptors (Rohrbough and Spitzer, 1999). Unlike developing brain areas, these spinal circuits have to function to produce reflexes and swimming from an early stage. It is therefore possible that the properties of the synapses arise "hard-wired" and independent of previous activity (Renger et al., 2001; Ziv and Garner, 2001), rather than following a path from NMDAR- to AMPAR-mediated excitation depending on activity. It will be fascinating to study whether the sensory pathway dlc interneurons that we defined in tadpoles are homologues of dorsal commissural interneurons defined by expression factors in the spinal cord of the developing mouse (Bermingham et al., 2001; Gowan et al., 2001).

\section{References}

Bermingham NA, Hassan BA, Wang VY, Fernandez M, Banfi S, Bellen HJ, Fritzsch B, Zoghbi HY (2001) Proprioceptor pathway development is dependent on Math1. Neuron 30:411-422.

Boothby KM, Roberts A (1995) Effects of site and strength of tactile stimulation on the swimming responses of Xenopus laevis embryos. J Zool 235:113-125.

Brodal P, Brodal A (1981) The olivocerebellar projection in the monkey. Experimental studies with the method of retrograde tracing of horseradish peroxidase. J Comp Neurol 201:375-393.

Clarke JDW, Roberts A (1984) Interneurones in the Xenopus embryo spinal cord: sensory excitation and activity during swimming. J Physiol (Lond) 354:345-362.

Clarke JDW, Hayes BP, Hunt SP, Roberts A (1984) Sensory physiology, anatomy and immunohistochemistry of Rohon-Beard neurones in embryos of Xenopus laevis. J Physiol (Lond) 348:511-525.

Dale N (1985) Reciprocal inhibitory interneurones in the Xenopus embryo spinal cord. J Physiol (Lond) 363:61-70.

Dale N, Roberts A (1985) Dual-component amino-acid-mediated synaptic potentials: excitatory drive for swimming in Xenopus embryos. J Physiol (Lond) 363:35-59.

Feldman DE, Knudsen EI (1998) Experience-dependent plasticity and the maturation of glutamatergic synapses. Neuron 20:1067-1071.

Fleshman JW, Rudomin P, Burke RE (1988) Supraspinal control of a shortlatency cutaneous pathway to hindlimb motoneurons. Exp Brain Res 69:449-459.

Gowan K, Helms AW, Hunsaker TL, Collisson T, Ebert PJ, Odom R, Johnson JE (2001) Crossinhibitory activities of Ngn1 and Math1 allow specification of distinct dorsal interneurons. Neuron 31:219-232.

Illert M, Lundberg A, Tanaka R (1976) Integration in descending motor pathways controlling the forelimb in the cat. 2. Convergence on neurones mediating disynaptic cortico-motoneuronal excitation. Exp Brain Res 26:521-540.

Kidokoro Y, Kubota K, Shuto S, Sumino R (1968) Reflex organization of cat masticatory muscles. J Neurophysiol 31:695-708.

Li WC, Perrins R, Soffe SR, Yoshida M, Walford A, Roberts A (2001) Defining classes of spinal interneuron and their axonal projections in hatchling Xenopus laevis tadpoles. J Comp Neurol 441:248-265.

Li WC, Soffe SR, Roberts A (2002) Spinal inhibitory neurons that modulatecutaneous sensory pathways during locomotion in a simple vertebrate. J Neurosci 22:10924-10934.

Reichert H, Rowell CH (1985) Integration of nonphaselocked exteroceptive information in the control of rhythmic flight in the locust. J Neurophysiol 53:1201-1218. 
Renger JJ, Egles C, Liu G (2001) A developmental switch in neurotransmitter flux enhances synaptic efficacy by affecting AMPA receptor activation. Neuron 29:469-484.

Roberts A, Sillar KT (1990) Characterization and function of spinal excitatory interneurons with commissural projections in Xenopus laevis embryos. Eur J Neurosci 2:1051-1062.

Roberts A, Walford A, Soffe SR, Yoshida M (1999) Motoneurons of the axial swimming muscles in hatchling Xenopus tadpoles: features, distribution, and central synapses. J Comp Neurol 411:472-486.

Roberts A, Hill NA, Hicks R (2000) Simple mechanisms organise orientation of escape swimming in embryos and hatchling tadpoles of Xenopus laevis. J Exp Biol 203:1869-1885.

Rohrbough J, Spitzer NC (1999) $\mathrm{Ca}^{2+}$-permeable AMPA receptors and spontaneous presynaptic transmitter release at developing excitatory spinal synapses. J Neurosci 19:8528-8541.

Sillar KT, Roberts A (1988a) Unmyelinated cutaneous afferent neurons activate two types of excitatory amino acid receptor in the spinal cord of Xenopus laevis embryos. J Neurosci 8:1350-1360.
Sillar KT, Roberts A (1988b) A neuronal mechanism for sensory gating during locomotion in a vertebrate. Nature 331:262-265.

Sillar KT, Roberts A (1992) The role of premotor interneurons in phasedependent modulation of a cutaneous reflex during swimming in Xenopus laevis embryos. J Neurosci 12:1647-1657.

Soffe SR (1993) Two distinct rhythmic motor patterns are driven by common premotor and motor neurons in a simple vertebrate spinal cord. J Neurosci 13:4456-4469.

Yoshida M, Roberts A, Soffe SR (1998) Axon projections of reciprocal inhibitory interneurons in the spinal cord of young Xenopus tadpoles and implications for the pattern of inhibition during swimming and struggling. J Comp Neurol 400:504-518.

Zhu JJ, Malinow R (2002) Acute versus chronic NMDA receptor blockade and synaptic AMPA receptor delivery. Nat Neurosci 5:513-514.

Ziv NE, Garner CC (2001) Principles of glutamatergic synapse formation: seeing the forest for the trees. Curr Opin Neurobiol 11: $536-543$. 